\title{
Morphological and Biochemical Analysis of Gallstones in Lahore, Pakistan
}

Anam Zahra, ${ }^{1}$ Muhammad Zain ul Abdin, ${ }^{2}$ Usman Ali Rahman, ${ }^{3}$ Shabbar Hussain Changazi, ${ }^{1}$ Samiullah Bhatti, ${ }^{1}$ Usman Ismat But, ${ }^{1}$ Mustansar Iqbal ${ }^{1}$

\begin{abstract}
Background: Gallstones are formed within the gallbladder or in biliary tract. They can be termed as cholesterol stones whose major constituent is cholesterol or pigment stones, which mainly are composed of bilirubin or there can be calcium and oxalate stones too.

Objective: To establish the composition of stones found within the gallbladder after cholecystectomy for symptomatic gallstones.

Methodology: This cross sectional study was conducted in Services Institute of Medical Sciences, Lahore, from $1^{\text {st }}$ August, 2017 to $30^{\text {th }}$ June, 2018, with a sample size of 200 consecutive patients of cholecystectomy for symptomatic gallstone. After examining the physical characteristics of the stones (color, number, shape and surface) were analyzed by semi- quantitative colorimetric analysis. Demographic data of patients, morphology of stones and the percentage different chemical components were noted on pre-established performa and analyzed by SPSS software version 22.

Results: The mean age of the 200 patients was $37.82 \pm 11.67$ years and $90 \%$ patients were female. Overall $10 \%$ cases had solitary stone while $90 \%$ cases had multiple stones and $25 \%$ patients had light brown stones, $30 \%$ had dark brown stones, $35 \%$ had gray stones and $10 \%$ had black stones. In this study, $85 \%$ cases had mixed stones whereas $15 \%$ had cholesterol stones. The mean percentages by weight of calcium, cholesterol, iron, phosphorus and bilirubin were $50 \pm$ $10.59 \%, 27.45 \pm 11.57 \%, 14.3 \pm 6.28 \%, 13.25 \pm 8.36 \%$ and $5.1 \pm 2.46 \%$ respectively.
\end{abstract}

Conclusion: It is concluded that gallstone disease is common in third decade of life and mixed stones is the most frequent variety of gallstones and calcium is the most abundant substrate of the gallstones.

Key words: Gallstones, Morphology, Chemical analysis

Article Citation: Zahra A, Abdin MZ, Rahman UA, Changazi SH. Bhatti S, Ismat U, Iqbal M. Morphological and Biochemical Analysis of Gallstones in Lahore, Pakistan. JSZMC 2020;11(1):12-16

\section{Introduction}

Gallstones have been known to mankind since long and were found in the gall bladders of Egyptian mummies. ${ }^{1}$ In majority of the cases gallstones are present in gall bladder but in some cases they can be present in biliary tract other than gallbladder. There are some cases in which intrahepatic stones are present along with gallstones but it has very less frequency. It is the most frequent disorder of gastrointestinal system with the prevalence of $10 \%$ to $15 \%$. $^{2}$ Gallbladder stones are of different sizes ranging from sand grain size to golf ball size. Females are affected more commonly with gall stone disease as compare to the males. ${ }^{3}$ It is more common in pregnancy due to increase in estrogen level which increases cholesterol secretion in bile. There are many etiologies for the formation of gallstones of which the common risk factors include female gender, ${ }^{4}$ obesity(fat), ${ }^{5}$ pregnancy, use of combined oral contraceptives, family history of stones, rapid weight loss and certain diseases like diabetes mellitus, ${ }^{6}$ liver disease and crohn disease. The pregnancy, however, was ruled out as a significant cause of gallstones in a study done in Germany by Walcher T. ${ }^{7}$

The chemical composition of gallstones varies in different parts of the world and changes over time due to dietary, environmental and demographic factors. ${ }^{8}$ Gallstones can be biochemically divided into three classes namely pure cholesterol gallbladder stones, pigment gallbladder stones and mixed gallbladder stones. Pure cholesterol stones are composed of pure cholesterol ( more than 50\%) and are of yellow-green colour. Mixed stones contain cholesterol, calcium salts, bile acids, phospholipids and bile pigments and pigmented gallstones are made of calcium bilirubinate (main ingredient). ${ }^{9}$ They are of brown or black colour depending upon

1.Services Institute of Medical Sciences, Department of General Surgery, Services Hospital, Lahore, Pakistan.

2.Gulab Devi Hospital, Lahore Pakistan.

3. Sahara Medical College, Narowal Pakistan.

Correspondence: Dr. Shabbar Hussain Changazi, Department of General Surgery, Services Institute of Medical Sciences, Lahore, Pakistan. 
their composition. The formation of gallstones is a complex mutifactorial process. Major factors that predispose the stone pathogenesis are: saturation of bile salts with cholesterol, crystal triglycerides nucleation, and impaired gall bladder function. ${ }^{10}$ Supersaturation of bile with cholesterol is the most important factor in the pathogenesis of gallstones. ${ }^{11}$ Cholesterol solubility in bile needs adequate amount of bile salt and phospholipids mainly phosphatidylcholine. If there is too much cholesterol or too little phospholipids and / or bile acid, complex vesicles are formed which lead to the nucleation of cholesterol crystals that result in stone formation. The production of cholesterol saturated bile, decreased amount of phospholipids, impaired gallbladder function and the resection of ileum were all associated with gallstone formation in gallstone formation. Pigmented gallbladder stones are formed when there is destruction of red blood cells which leads to excretion of large amount of bilirubin in the bile. In diseases like liver cirrhosis and chronic hemolytic conditions black pigmented stones are formed. The chronic hemolytic diseases include thalasemias, sickle cell disease and hereditary spherocytosis. ${ }^{11}$

In these diseases bilirubin secretion is increased due to increase in hemolysis of red blood cells. Infection is another cause of pigmented stones. Stones that originates in bile duct are known as primary bile duct stones. These are usually brown pigmented stones that results due to infection even after cholecystectomy. $\beta$-glucuronidases is released in biliary system by bacteria. This hydrolyze glucuronic acid from conjugated bilirubin. This results in increase in unconjugated bilirubin. This unconjugated billirubin then precipitates as its calcium salts. These brown pigment stones of the bile ducts are more common among Asians. Another associated factor is decreased biliary secretory Immunogloblin A (IgA.). ${ }^{12}$

Gallstones disease is quite common in Pakistan especially in females and contributes to a significant load of patients presenting to emergency departments and in elective settings. Although data about prevalence of gallstones is insufficient in Pakistan, a previous study conducted in province Sindh reported an overall incidence of $9 \%$. There has been a rising pattern in recent years in the number of gallstones cases in Pakistan. ${ }^{13,14}$ This increasing incidence of gallstones in Pakistan with its various epidemiological determinants convinced us to conduct this study to assess the chemical composition examination of gallstones and this study reveals morphology of gallstones and major constituents and substrate of gallstones in local setting.

\section{Methodology}

This was a cross-sectional study conducted in a tertiary care hospital i-e. Services Institute of Medical Sciences Lahore, from $1^{\text {st }}$ August, 2017 to $30^{\text {th }}$ June, 2018. A total of 200 consecutive patients, who underwent cholecystectomy for symptomatic gallstones, were included in study. Patients of both gender with age 15 years and above presenting to surgical outdoor with symptomatic gallstones, were included in the study. Exclusion criterion was also defined as patients who developed gallstones after bariatric surgery. All stones removed during operation were placed on sterile gauze to air dry and transferred into sterile jar after examining the macroscopic characteristics of stones, such as color, number, shape, texture, and cross section. The specimen was then sent to laboratory for biochemical analysis after tagging specimen with name and age and sex.

The size of the stones was determined by measuring the largest diameter of single stone or deriving the average for the several stones. Percentage of cholesterol, calcium, phosphorus, iron and bilirubin in gall bladder stones, were determined by using semi- quantitative colorimetric analysis. For this purpose commercially available biolabo reagents kit, France was used. Demographic data of patients, morphology of stones and the percentage by weight of cholesterol, calcium, phosphorous, iron and bilirubin in the stones, were noted on pre-established performa. Ethical approval was sought from Ethical Committee of Institute. Data was analyzed by using SPSS software version 22.

\section{Results}

A total of 200 people were enrolled in this study. The mean age of the patients was $37.82 \pm 11.67$ years and ranged from 21 years to 75 years. Out of total 200 patients, $180(90 \%)$ patients were female and 20 $(10 \%)$ patients were males and $60(30 \%)$ cases had small stones (size less than $1 \mathrm{~cm}), 120(60 \%)$ had 
medium sized stones (size 1 to $3 \mathrm{~cm}$ ) and $20(10 \%)$ cases had large stones (size greater than $3 \mathrm{~cm}$ ). in this study, $20(10 \%)$ cases had solitary stone, 120 $(60 \%)$ had 2 to 4 stones while $60(30 \%)$ cases had more than 4 stones. It was noted that $50(25 \%)$ patients had light brown stones, $60(30 \%)$ had dark brown stones, 70 (35\%) had gray stones and $20(10 \%)$ had black stones.

It was found that $160(80 \%)$ gallbladder stones had smooth surface while $40(20 \%)$ gallbladder stones had irregular surface whereas, $140(70 \%)$ cases had irregular shape of stones while $60(30 \%)$ had rounded stones and $170(85 \%)$ cases had mixed stones whereas, $30(15 \%)$ had cholesterol stones. (Table I)

The mean percentage of calcium by weight in gallstones was $40.50 \pm 10.59 \%$, percentage of cholesterol was $27.45 \pm 11.57 \%$, percentage of iron was $14.3 \pm 6.28 \%$, percentage of phosphorus was $13.25 \pm 8.36 \%$ and percentage of bilirubin was $5.1 \pm 2.46 \%$. (Table II)

Table I: Morphology of gallstones

\begin{tabular}{|c|c|c|}
\hline Morphology & Frequency & $\begin{array}{l}\text { Percentage } \\
(\%)\end{array}$ \\
\hline \multicolumn{3}{|l|}{ Size of stones } \\
\hline Small(less than $1 \mathrm{~cm}$ ) & 60 & 30 \\
\hline Medium(1 to $3 \mathrm{~cm})$ & 120 & 60 \\
\hline Large (greater than $3 \mathrm{~cm}$ ) & 20 & 10 \\
\hline \multicolumn{3}{|l|}{ Number of stones } \\
\hline 1 & 20 & 10 \\
\hline 2 to 4 & 120 & 60 \\
\hline More than 4 & 60 & 30 \\
\hline \multicolumn{3}{|l|}{ Surface of stones } \\
\hline Rough & 40 & 20 \\
\hline Smooth & 160 & 80 \\
\hline \multicolumn{3}{|l|}{ Shape of stones } \\
\hline Irregular & 140 & 70 \\
\hline Smooth & 60 & 30 \\
\hline \multicolumn{3}{|l|}{ Type of stones } \\
\hline Cholesterol stones & 30 & 15 \\
\hline Mixed stones & 170 & 85 \\
\hline
\end{tabular}

Table II: Chemical Composition of Gallstones

\begin{tabular}{|l|l|l|}
\hline Substrate of stones & $\begin{array}{l}\text { Mean } \\
\text { percentage } \\
(\%)\end{array}$ & SD \\
\hline Calcium & 40.50 & 10.59 \\
\hline Cholesterol & 27.45 & 11.57 \\
\hline Iron & 14.3 & 6.28 \\
\hline Phosphorus & 13.25 & 8.36 \\
\hline Bilirubin & 5.1 & 2.46 \\
\hline
\end{tabular}

\section{Discussion}

Gallstone disease is a common disorder of the gastrointestinal tract, having a prevalence of $10 \%-15 \%$ and an incidence of $1.4 \%$ per year in the adult population. ${ }^{2}$ Pathogenesis of gallstones is multifactorial and detection of chemical composition of gallstones is primarily important to identify their mechanism of formation. Of the three types of gallstones, pigment and mixed cholesterol stones were the commonest types identified in this study. Further, majority of gallstones analyzed in this study were composed of multiple chemical compounds namely calcium, cholesterol, iron, phosphorus and bilirubin. This highlights the importance of exploring the aetiology for calcium salt and cholesterol precipitation in bile in these patients.

In this study most of the patients with gallstone disease were in their third decade of life with mean age of 37.82 years. Angwafo at $\mathrm{el}^{14}$ showed mean age of 44.9 years with age ranged from 27 and 73 years. Jarrar BM at $\mathrm{el}^{15}$ described mean age for gallstone disease to be $46.1 \pm 11.6$ years. This study showed that mean age was slightly younger than these other studies. This pattern can be attributed to type of food and its quality commonly used in our local setting that is rich in carbohydrates and lipids. Our diet contains more animal fat of poor quality which may result in increased incidence of gallbladder stones in early age. Pregnancy is one of the factor in development of gallstones. In our population we have early marriages which lead to early pregnancy in younger age group resulting in gallbladder stone formation in younger age group.

In the study, the gallstone disease was more prevalent in females as compared to males with 10:1 female: male ratio. Other researchers in their studies also observed similar results. ${ }^{14,15,16}$ These results with 
female preponderance in gallstone disease can attributed to females sex hormones coupled with obesity, repeated pregnancy, sedentary life style, use of contraceptive pills and consumption of lipid rich food in the local region. This difference in ratio decreases with increase in age. In postmenopausal stage this difference is very less due to lack of estrogen level which mainly contribute in formation of gallstones due to increase in cholesterol secretion in bile.

In our study most common (60\%) stones were of medium size (1 to $3 \mathrm{~cm}$ ) followed by $(30 \%)$ small stones (less than $1 \mathrm{~cm}$ ). Similarly in most patients $(90 \%)$ more than one stone was present. These both findings are in correlation to other national and international studies. The size and number of stones both are directly related to age of the patient. As the age increases both size and number increases. ${ }^{4}$

In the present study $170(85 \%)$ had mixed type of gallstones whereas $30(15 \%)$ patients had cholesterol gallstones. Weerakoon $\mathrm{H}$ et $\mathrm{al}^{16}$ and Afridi $\mathrm{R}$ et $\mathrm{al}^{17}$ in their study showed that pigmented stones were the most frequent type of stones accounting upto $50.67 \%$ and $54 \%$ respectively. A study conducted by Channa NA at $\mathrm{el}^{18}$ revealed that cholesterol stones were the common form of gallstones with $74 \%$ of total cases. Similar results were also reported in other studies. ${ }^{15,19}$ The prevalence of mixed stones in this study can be due to multifactorial including environmental factors, consumption of type of food, genetic differences and metabolic errors. In western countries and Pakistan majority of gallstones are pure cholesterol stones or mixed stones with increase amount of cholesterol levels in them.

In the current study calcium was the main substrate of the gallstones with mean percentage of $40.50 \%$. The second common ingredient of gallstones was cholesterol with mean percentage of $27.45 \%$. Iron accounted for $14 \%$ while phosphorus weighted $13.25 \%$ of total gallstone composition. Bilirubin was the least frequent ingredient of gallstones. Schafmayer $\mathrm{C}$ et $\mathrm{al}^{20}$ in a study of gallstone analysis found that cholesterol was the main ingredient of gallstones accounted for $95 \%$ of total composition followed by calcium and then bilirubinate. A study conducted in Saudi Arabia also concluded that cholesterol was the major component (83\%) of the gallstones, followed by calcium, bilirubin, and carbonate. ${ }^{16}$ Similar results were also described by other researchers. ${ }^{17,} 18,21,22$ However, some results evinced that bilirubinate was the common substrate of gallstones. ${ }^{22,23}$ In our study calcium was the most common ingredient followed by cholesterol in gallbladder stones. Most common cause of calcium stones identified is recurrent infection and biliary obstruction. This difference of increased calcium instead of cholesterol is due to more incidences of gallbladder and biliary duct infections in our population.

\section{Conclusion}

It is concluded that gallstone disease is common in third decade of life and more common among female as compared to male. It is also noted that mixed type of stones are the most frequent type of gallstones and calcium is the most abundant substrate in the gallstone composition which is contrary to other national and international studies. More studies with increased sample size is recommended to proceed forward.

Authors Contribution: AZ: Conception of work and Design of Work. MZA: Analysis of data and drafting. UAR: Drafting and Revising. SHC: Acquisition \& analysis. SB: Analysis of data and drafting. UIB: Analysis of data and drafting. MI: Analysis of data and drafting.

All the authors gave final approval for publication and agreed to be accountable for all aspect of work.

\section{Conflict of Interest: None \\ Sources of Funding: Self}

\section{References}

1. Berci G. Historical overview of surgical treatment of biliary stone disease. In: MacFadyen BV, ArreguiM, Eubanks S, Olsen DO, Peters JH, Soper NJ, editors. Laparoscopic surgery of the abdomen. New York (NY): Springer; 2004. p. 139-142.

2. Batajoo H, Hazra NK. Analysis of serum lipid profile in cholelithiasis patients. J Nepal Health Res Counc 2013 Jan;11(23): 53-5

3. Attili AF, Carulli N, Roda E. Epidemiology of gallstone disease in Italy: prevalence data of the Multicenter Italian Study on Cholelithiasis (M.I.COL.). Am J Epidemiol 1995; 141:158.

4. Keane P, Colwell D, Baer HP. Effects of age, gender and female sex hormones upon contractility of the human gallbladder in vitro. SurgGynecolObstet1986; 163: 55560. 
5. Amaral JF, Thompson WR. Gallbladder disease in the morbidly obese. Am J Surg 1985; 149: 551-7.

6. Jorgensen T. Gall stones in a Danish population: relation to weight, physical activity, smoking, coffee consumption, and diabetes mellitus. Gut 1989; 30: 2834.

7. Walcher T, Haenle MM, Kron M, Hay B, Mason RA, Von Schmiesing AF, et al. World J Gastroenterol 2005 Nov 21; 11(43): 6800-6

8. Jensen KH, Jorgensen T. Incidence of gallstones in Danish population. Gastroenterology 1991; 100(3): 790-794.

9. Van Erpecum KJ. Pathogenesis of cholesterol and pigment gallstones: An update. Clin Res Hepatol Gastroenterol 2011;35(4): 281-87.

10. Dowling RH. Review: Pathogenesis of gallstones. Aliment Pharmacol Ther 2000;14 (Suppl 2): 39-47

11. Ko CW and Lee SP. Gallstone formation. Local factors. GastroenterolClin North Am 1999; 28(1): 99-115.

12. Channa NA, Khand FD, Bhanger MI, Laghari MH. Surgical incidence of Cholelithiasis in Hyderabad and adjoining areas(Pakistan). Pak J Med Sci 2004; 20: 137.

13. Khand FD, Ansari AF, Khand TU. Cholelithiasis in Southern Sindh (Pakistan) incidence and composition of gallstones. Specialist Pak J Med Sci 1997; 13(3): 263-70.

14. Angwafo III FF, Takongmo S, Griffith D. Determination of chemical composition of gall bladder stones: basis for treatment strategies in patients from Yaounde, Cameroon. World Journal of Gastroent 2004 Jan $15 ; 10(2): 303$.

15. Jarrar BM, Al-Rowaili MA. Chemical composition of gallstones from Al-jouf province of saudiarabia. The Malaysian J of Med Scienc 2011 Apr; 18(2): 47.
16. Weerakoon H, Navaratne A, Ranasinghe S, Sivakanesan R, Galketiya KB, Rosairo S. Chemical characterization of gallstones: an approach to explore the aetiopathogenesis of gallstone disease in Sri Lanka. PloS one 2015 Apr 8; 10(4): e0121537.

17. Afridi R, Afridi FG, Batool Z. Patho-morphologic classes of gallstones, their frequency and etiological factors in Peshawar Pakistan. Journal of Rehman Medical Institute 2015 Oct 17;1(1).

18. Channa NA, Khand FD, Khand TU, Leghari MH, Memon AN. Analysis of human gallstones by Fourier Transform Infrared (FTIR). Pakistan Journal of Med Scienc 2007 Jul $1 ; 23(4): 546$.

19. Bowen JC, Brenner HI, Ferrante WA, Maule WF. Gallstone disease. Pathophysiology, epidemiology, natural history, and treatment options. The Medical Clinics of North America 1992 Sep; 76(5): 1143-57.

20. Schafmayer C, Hartleb J, Tepel J, Albers S, Freitag S, Völzke H, Buch S, Seeger M, Timm B, Kremer B, Fölsch UR. Predictors of gallstone composition in 1025 symptomatic gallstones from Northern Germany. BMC gastroenterology $2006 \mathrm{Dec} ; 6(1): 36$.

21. Jaraari A, Jagannadharao P, Patil T, Hai A, Awamy H, El Saeity S, Abdel Kafi E, El-Hemri M, Tayesh M. Quantitative analysis of gallstones in Libyan patients. Libyan Journal of Medicine 2010 Jan 1; 5(1): 4627

22. Mokhtar AMA. Cholelithiasis in the western region of Saudi Arabia. Est African Medical J 1990; 67(4): 286-290.

23. Chandran P, Kuchhal NK, Garg P, Pundir CS. An extended chemical analysis of gallstone. Indian $\mathrm{J}$ Clin Biochem 2007; 22(2): 145-150 